\title{
Application of the Virtual Information Fabric Infrastructure (VIFI) to Building Performance Simulations
}

\author{
Yimin $\mathrm{Zhu}^{1 *}$, Yong Tao ${ }^{2}$ and William J Tolone ${ }^{3}$ \\ ${ }^{1}$ Department of Construction Management, Louisiana State University, Baton Rouge, LA, USA \\ ${ }^{2}$ Department of Mechanical Engineering, Cleveland State University, Cleveland, $\mathrm{OH}$, USA \\ ${ }^{3}$ College of Computing and Informatics, University of North Carolina at Charlotte, Charlotte, NC, USA
}

*Corresponding author: Yimin Zhu, Department of Construction Management, Louisiana State University, Baton Rouge, LA, USA.

Received Date: October 25, 2019

Published Date: November 06, 2019

\begin{abstract}
Although contemporary information and computer technologies enable researchers, designers, and engineers to collect large amounts of data, current approaches for sharing data remain limited by concerns such as interoperability, shareability, data size, transport costs, and privacy. These concerns often prevent or confound the development of reliable and accurate building performance simulations. In an effort to overcome these concerns, we envisioned a complementary approach, called the Virtual Information Fabric Infrastructure (VIFI) approach, where data owners share distributed, fragmented data in a manner that does not require the movement of raw data and data users utilize these data for analysis by transporting analytic computation to the data. The VIFI approach presents new opportunities to conduct building performance simulations. Through two case studies, we demonstrated a new computational framework based on VIFI to support an open and collaborative cyberinfrastructure for building performance simulations. Such a computational framework represents a system view toward building performance simulations, in which essential components of simulations are coherently integrated.
\end{abstract}

\section{Introduction}

During the design and engineering phase of a new building, computer-based simulations are commonly used to understand, analyze, and predict the performance of the new building such as energy, water, thermal comfort, indoor air quality, lighting, and acoustics. Often, such simulations require two indispensable ingredients in order to produce reliable and accurate simulations: i) a significant amount of empirical data for modeling, calibration, and validation; and, ii) insightful knowledge of anticipated humanbuilding interactions for the building under design. Empirical data are conventional sources for creating simulation models. However, since each building is unique, simulations based solely on empirical data are often not able to address fully the unique context of a new building. In addition, even though contemporary technologies enable researchers, designers, and engineers to collect large amounts of data for various types of buildings, access to such data across organization and ownership boundaries often is challenging due to concerns such as interoperability, shareability, data size, transport costs, and privacy. Therefore, the identification of effective methods to combine these two ingredients (distributed raw data and understanding human-building interactions during design) for building performance simulations remains an open challenge within the building design and engineering communities. Recent advances in computing technologies and data analysis methods offer new opportunities to improve computer-based, new building simulations that otherwise remain underexplored by the broader research community. For example, we are conducting new building simulation experiments within an open computing infrastructure based on the concept of dynamic data-driven application systems (DDDAS). This infrastructure, called the Virtual Information Fabric Infrastructure (VIFI) [1], combines cyberinfrastructure data, data science methods, and immersive computing capabilities in a manner that presents a new approach for conducting building performance simulations. The VIFI approach is complementary to the conventional data-centric sharing strategy, which focuses on transporting raw data for subsequent analyses. Instead, the 
VIFI approach focuses on transporting data analytics (rather than raw data) and, thus, enables analysis when raw data are not easily transported or shared. Under the VIFI approach, raw data do not leave the site of the data owner, nor are data directly accessed by data users.

\section{Cases}

We first implemented a case to show the applicability of the VIFI approach to support building performance simulations [2] (Figure 1).

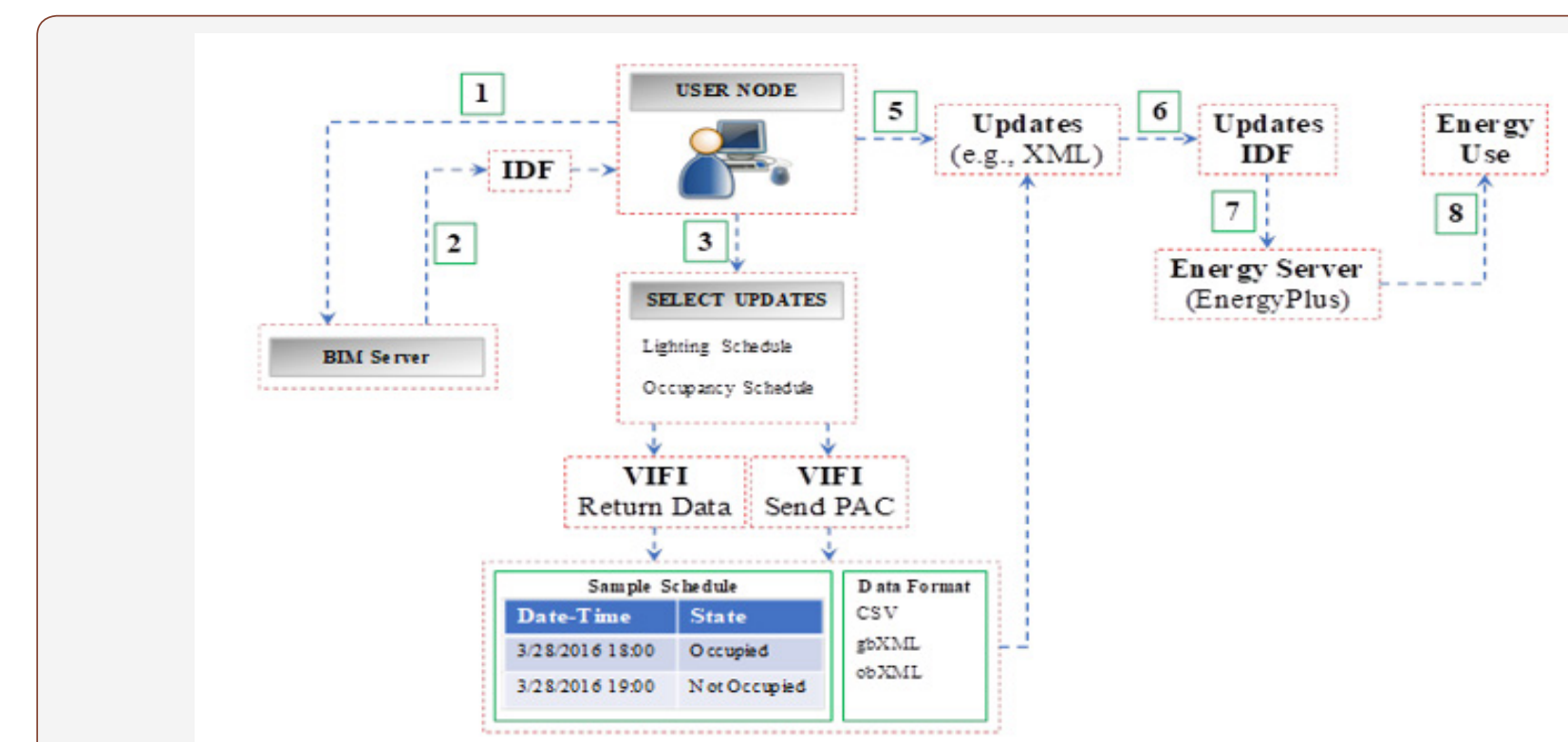

Figure 1: Modeling Building Energy Performance Using Distributed, Measured Occupancy Data.

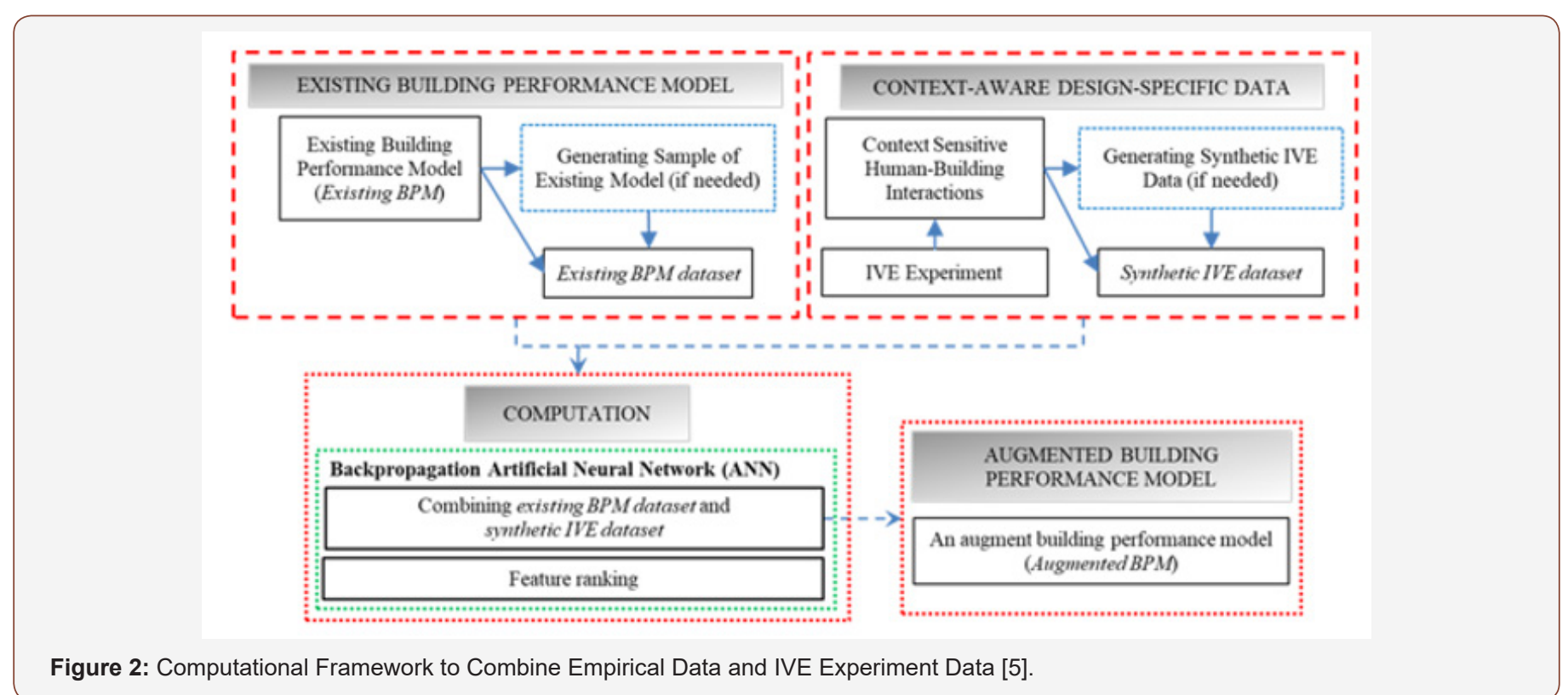

The building simulation tool, Energy Plus, was used to simulate the small-sized commercial office building reference model provided by the US Department of Energy. Through the case study, we successfully integrated empirical artificial light use and occupancy data with an analytic model, the energy simulation of the reference model. The raw artificial light use, and occupancy schedule data were not shared directly or transported from the data source to the data users. Rather, the raw data were transformed into a lighting schedule and an occupancy schedule through analysis performed at the data source. The schedules, compatible with the input data file (IDF) format of Energy Plus, were transported to a remote server for simulations. In addition, the case study revealed the possibility of integration between VIFI and building information modeling (BIM) tools. If BIM represents the conventional datacentric sharing strategy, the integration shows that the VIFI approach can work with the conventional data sharing strategy to achieve their common goal. While VIFI directly addresses the challenge to provide the first ingredient (i.e., distributed raw data) for building performance simulations, immersive computing has the potential to provide solutions for the second ingredient (i.e., understanding human-building interactions during design). This is because immersive computing allows designers to observe and analyze human-building interactions for buildings under design [3]. Accordingly, we implemented another case study, a computational 
framework combining an existing building performance model, the Hunt model [4] (i.e., an analytic model of artificial light use) with context-aware design-specific data collected from immersive virtual environments (IVEs). The computational framework uses a machine-learning algorithm, i.e., artificial neural networks (ANNs) [5], to combine the Hunt model and design specific data from IVE experiments (e.g., turn on/off artificial lights at different illuminance levels and for different events) (Figure 2).

The results of the case study show that the accuracy of the existing light use simulation model can be significantly improved compared with data collected from the actual office. Although this case study is not directly deployed on VIFI, the first case has already demonstrated the capability of VIFI to integrate empirical data and analytic models.

\section{Implications to Future Research and Development}

Based on the two cases, we envision a new, VIFI-based computational framework for building performance simulations, an open and collaborative cyberinfrastructure that involves the operational data of existing buildings, experiment data relevant to specific design conditions, as well as other computing and computational components such as machine-learning algorithms (Figure 3).

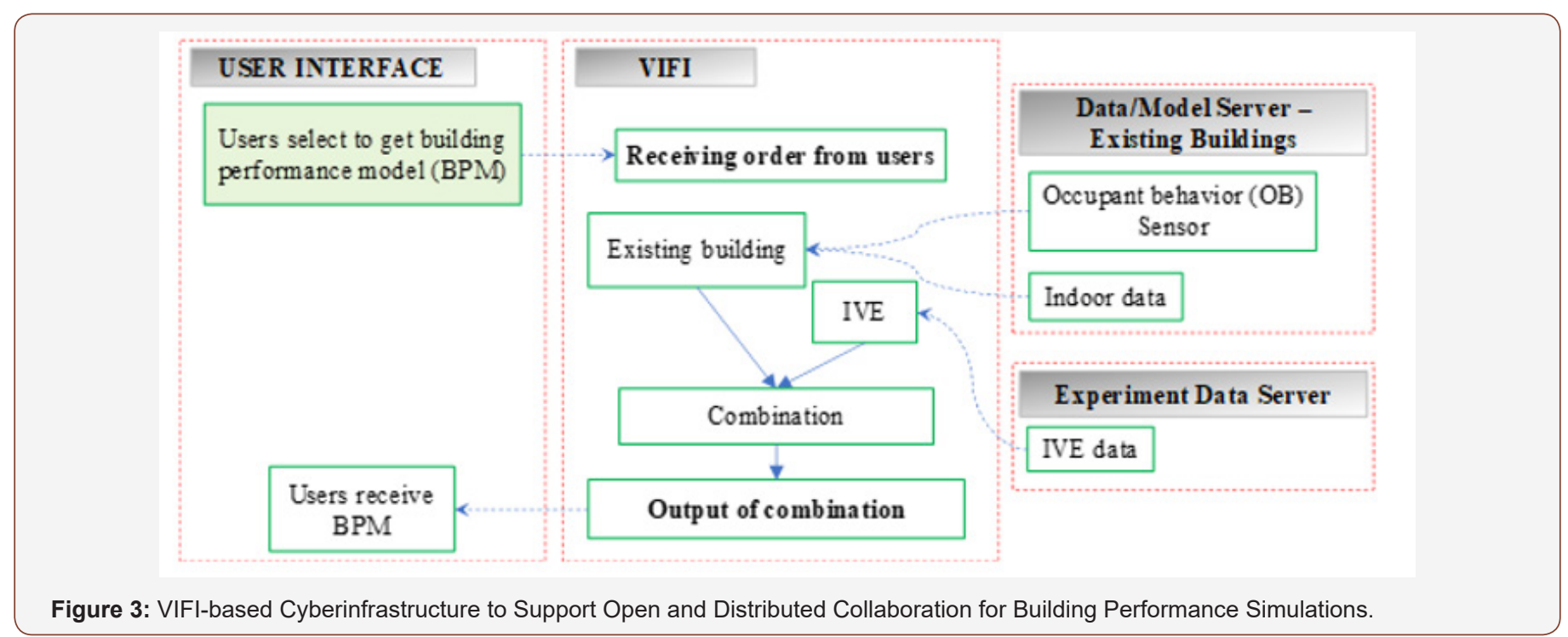

Such a framework represents a system view towards building performance simulations, where essential components of simulations are coherently integrated. In summary, our studies show that the combination of VIFI, existing building data or models, and experiment data (e.g., using IVEs) presents an unexplored opportunity for fundamentally changing how we perform building simulations in the future and significantly improving simulation results by incorporating context-specific human-building interactions. Certainly, there are limitations associated with the framework such as the reliability of data collected from IVE experiments.

\section{Acknowledgement}

This paper was partially supported by the U.S. National Science Foundation Award \#1640818. Any opinions, findings, and conclusions or recommendations expressed in this material are those of the author(s) and do not necessarily reflect the views of the National Science Foundation.

\section{Conflict of Interest}

No conflict of interest.

\section{References}

1. Elshambakey M, Mohamed K, William JT, Sreyasee Das Bhattacharjee, Huikyo L, et al. (2017) Towards a distributed infrastructure for datadriven discoveries \& analysis. 2017 IEEE Smart World, Ubiquitous Intell. Comput. Adv. Trust. Comput. Scalable Comput. Commun. Cloud Big Data Comput. Internet People Smart City Innov, pp.1-8.

2. Karaguzel OT, Mohammed E, Yimin Z, Tianzhen H, William JT, et al. (2019) Open Computing Infrastructure for Sharing Data Analytics to Support Building Energy Simulations. J Comput Civ Eng 33: 04019037.

3. Heydarian A, Pantazis E, Carneiro JP, Gerber D, Becerik-Gerber B (2016) Lights, building, action: Impact of default lighting settings on occupant behaviour. J Environ Psychol 48: 212-223.

4. Hunt DRG (1979) The use of artificial lighting in relation to daylight levels and occupancy. Build. Environ 14: 21-33.

5. Chokwitthaya C, Zhu Y, Dibiano R, Mukhopadhyay S (2019) Combining context-aware design-specific data and building performance models to improve building performance predictions during design. Autom Constr 107: 102917. 